\title{
The influence of optical aberrations on the magnitude of the Poggendorff illusion
}

STANLEY COREN 1

NEW SCHOOL FOR SOCIAL RESEARCH

\begin{abstract}
The amount of blurring of the retinal image can be reduced by proper selection of an artificial pupil and a chromatic filter. Reduction of the amount of blurring due to optical aberrations by viewing through a $1-\mathrm{mm}$ artificial pupil and an interference filter in the eye, results in a concomitant reduction in the magnitude of the Poggendorff illusion. The magnitude of the reduction is smaller, however, than would be expected if the illusion was predominantly determined by blur due to optical aberration.
\end{abstract}

Recently there has been a revival of interest in a theory of visual illusions first proposed by Einthoven (reviewed in Titchener, 1901) and restated by Chiang (1968) in more contemporary terms. This theory is based on the fact that the actual distribution of energy on the retina is quite different from the external stimulus distribution presented to the eye. Numerous intrinsic optical aberrations tend to blur the image and shift the relative maximum of the stimulus distribution imaged on the retina. The subjective locus of a contour corresponds to the peak of the resultant distribution. Chiang has presented a geometrical analysis of the resultant energy distribution that suggests that such shifts in the maxima of the distribution may account for several of the classical visual-geometric illusions involving intersecting lines, such as the Poggendorff. This hypothesis is directly testable since the major sources of blur on the retina are controllable, and such a test is presented below.

\section{METHOD}

The major sources of blurring of the image on the retina are chromatic aberration, spherical aberration, diffraction, and uncorrected refractive errors in the eye (Ludvigh, 1953; Sheard, 1926; Ames \& Proctor, 1921). Table 1 lists the approximate diameter of the blur disc for a 4-mm pupil, expressed in seconds of arc at the nodal point of the eye for a theoretical point source. It is clear from this table that the principal sources of blurring are spherical and chromatic aberration. If we present the same theoretical point source through an artificial pupil of $1-\mathrm{mm}$ diam and limit the wavelengths passing through the pupil by use of an interference filter that only passes wavelengths of light between 520 and 538 millimicrons as shown in Table 1 , we significantly decrease all of the sources of blurring except for diffraction, which is increased. It is hard to know whether the most important variable is the size of the maximal blur circle or the total of all of the blur circles. If we take the maximal blurring

Table 1

Approximate Diameter of the Blur Disc Expressed in Seconds of Arc Subtended at the Nodal Point of the Eye.

\begin{tabular}{lcc}
\hline & $4 \mathrm{~mm}$ pupil & $1 \mathrm{~mm}$ pupil with filter \\
\hline Spherical aberration & $432 \mathrm{sec}$ & $30 \mathrm{sec}$ \\
Chromatic aberration & $216 \mathrm{sec}$ & $13 \mathrm{sec}$ \\
Diffraction & $34 \mathrm{sec}$ & $126 \mathrm{sec}$ \\
Assumed uncorrected & & $13 \mathrm{sec}$ \\
refractive error of .0625D $54 \mathrm{sec}$ & $182 \mathrm{sec}$ \\
\cline { 2 - 3 } Total & $736 \mathrm{sec}$ &
\end{tabular}
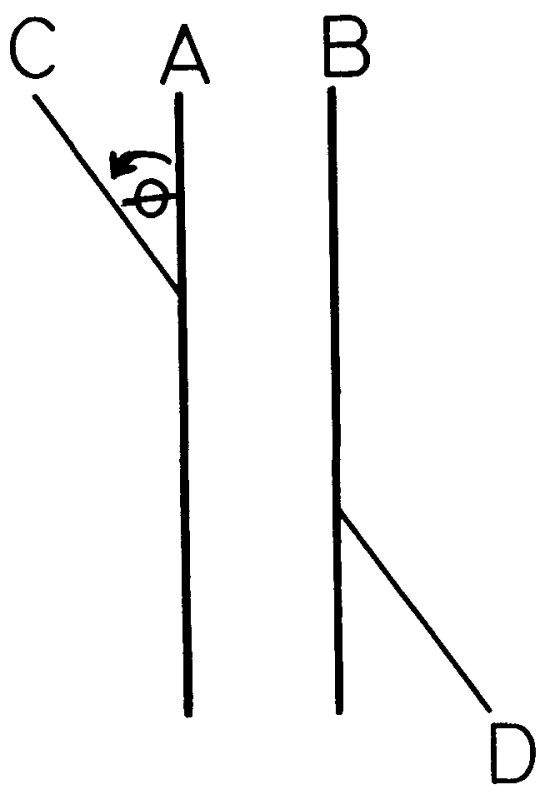

Fig. 1. The Poggendorff illusion.

from any one source, the $1-\mathrm{mm}$ pupil and filter provide only $3 / 10$ as much blur as the 4-mm pupil. If we take the total, we reduce the blur to $1 / 4$.

\section{Apparatus}

Figure 1 shows the Poggendorff illusion, which was the illusion used in this experiment. The lines $A$ and $B$ were $11 \mathrm{~cm}$ in length and separated by $5 \mathrm{~cm}$. They were formed from black chart tape $4 \mathrm{~mm}$ wide. Line $C$ was $9.5 \mathrm{~cm}$ long and Line D was $8.5 \mathrm{~cm}$. They formed an angle $\theta=30 \mathrm{deg}$ with the vertical lines. Line $\mathrm{D}$ was mounted on a piece of clear Plexiglas that could be adjusted up and down along a vertical track. The height at which $D$ was set could be read in millimeters by $\mathbf{E}$, from a scale affixed to the track. S stood approximately at arm's length from the apparatus. Two artificial pupils were employed. One was $4 \mathrm{~mm}$ in diam, the other was $1 \mathrm{~mm}$ in diam. The $1-\mathrm{mm}$ pupil also contained an interference filter that passed light of wavelengths 520 to $538 \mathrm{mu}$. Lighting was adjusted so that under each condition the retinal illumination was approximately 220 trolands.

\section{Subjects}

Twelve volunteers were recruited from the New School for Social Research. Six were male and six female. All were naive as to the purpose of the experiment.

\section{Procedure}

Each S served under both viewing conditions. Half of the Ss viewed the figure with the 4-mm pupil first and half with the 1-mm pupil and filter. Each $S$ made two settings on the illusion 
figure under each viewing condition. Settings were counterbalanced for starting position. Ss were instructed to set Line D so that it appeared to be a continuation of Line $C$.

\section{RESULTS AND DISCUSSION}

If the Poggendorff illusion is determined by the blurred distribution of energy on the retina, due to optical aberrations, then viewing the illusion figure through the $1-\mathrm{mm}$ pupil with the narrow band interference filter should result in a decrease in the magnitude of the illusion over the condition in which $S$ views the figure through a 4-mm pupil with no filter, because of the significant reduction in the amount of blurring. The mean illusion magnitude for the 4-mm pupil viewing condition is $23.94 \mathrm{~mm}$ while for the $1-\mathrm{mm}$ pupil it is $20.20 \mathrm{~mm}$. This difference is significant when tested by a sign test with $\mathrm{p}<0.05$. This result confirms the involvement of blurring of the retinal image in the formation of the illusion.

When we look at the magnitude of this reduction of the Poggendorff illusion, however, some difficulties become apparent. If we assume any symmetrical distribution of energy on the retina and then decrease its spread to $1 / \mathrm{N}$ of the original spread of energy, then the magnitude of the illusion should be reduced to $1 / \mathrm{Nth}$ of the original illusion. This can be easily demonstrated geometrically along the lines suggested by Chiang (1968) and Pressey and den Heyer (1968). In the 1-mm pupil condition the blur is reduced to $1 / 4$ if we assume that total blur is the determinant factor, and to approximately $3 / 10$ if the largest single blur circle is the determinant factor. Thus we would expect approximately a $70 \%$ reduction in the magnitude of the illusion under the low blur conditions. Instead of a $70 \%$ reduction, however, the decrease in the illusion magnitude is only $15.6 \%$, which is significantly less than $70 \%$ with $\mathrm{p}<0.01$.

Thus it becomes clear that although blurring due to optical aberrations in the eye certainly plays a significant contributory role in the formation of the Poggendorff jllusion, it is clearly not the primary determining factor as has been suggested by Chiang.

\section{REFERENCES}

AMES, A., \& PROCTOR, C. A. Diptrics of the eye. Journal of the Optical Society of America, 1921, 5, 22-35.

CHIANG, $C$. A new theory to explain geometrical illusions produced by crossing lines. Perception \& Psychophysics, 1968, 3, 174-176.

LUDVIGH, E. Perception and contour. U.S. Naval School of Medicine, 1953, Project No. NM 001075.01 .04 , Report No. 4.

PRESSEY, A. W., \& den HEYER, K. Observations on Chiang's "new" theory of geometrical illusions. Perception \& Psychophysics, 1968, 4, 313-314.

SHEARD, C. S. The chromatic aberration of the eye, the chromatic variations in the interval of the sturm and allied phenomena as determined by a subjective method of skiascopy. Journal of the Optical Society of America, 1926, 12, 79-82.

TITCHENER, E. G. Experimental psychology. Vol. I, Part II. New York: MacMillan Co., 1901.

\section{NOTE}

1. Address: Department of Psychology, New School for Social Research West 12 th Street, New York, New York 10011.

(Received for publication February 12, 1969.) 